\title{
Validity of Signal Peptide-CUB-EGF Domain-containing Protein-1 (SCUBE-1) in the Diagnosis of Aortic Dissection
}

\author{
Adem Çakır $1, \star$, Umut Payza ${ }^{2}$, Saliha Aksun ${ }^{3}$, Ahmet Kayalı², Zeynep Karakaya ${ }^{2}$, \\ Fatih Esad Topal ${ }^{2}$
}

\begin{abstract}
${ }^{1}$ Department of Emergency Medicine, The Ministry of Health Basaksehir City Hospital, Istanbul, Turkey

${ }^{2}$ Department of Emergency Medicine, Izmir Katip Çelebi University Atatürk Training and Research Hospital, Izmir, Turkey

${ }^{3}$ Departmant of Biochemistry, Izmir Katip Celebi University Atatürk Traning and Researh Hospital, Izmir, Turkey
\end{abstract}

*Correspondence

dr.ademcakir@hotmail.com

(Adem Çakır)

\begin{abstract}
Introduction: Diagnosing aortic dissection (AD) in emergency services still represents a challenging issue as it may manifest not only well-known clinical findings such as sudden onset and severe chest pain but also atypical findings similar to ST elevation myocardial infarction or renal colic. Contrast-enhanced imaging investigations, which are expensive and risky due to possible complications, are necessary for diagnosis. Ultrasonography is not always reliable, which necessitates noninvasive diagnostic tests to support a clinical suspicion. Signal peptide-CUB (complement C1r/C1s, Uegf, and Bmp1)-EGF (epidermal growth factor) domain-containing protein 1 (SCUBE-1) is a cell surface protein produced during embryogenesis. This study was conducted to compare the levels of this novel biomarker between patients with $\mathrm{AD}$ and healthy volunteers. Methods: This prospective study was conducted on 20 patients diagnosed with AD using contrast-enhanced thoracoabdominal computed tomography angiography. Average agematched 20 healthy subjects as a control group were included as a reference for biochemical parameters. Results: The mean SCUBE-1 levels were significantly higher in patients with $\mathrm{AD}[24.51( \pm 3.01) \mathrm{ng} / \mathrm{dL}]$ than in the control group $[12.11( \pm$ $5.31) \mathrm{ng} / \mathrm{dL}](\mathrm{p}<0.001)$. Receiver-operating characteristic (ROC) curve was plotted to analyze the specificity and sensitivity of AD diagnosis (with 95\% confidence intervals), which revealed $95 \%$ sensitivity and $76 \%$ specificity when the SCUBE-1 level was $>19.75 \mathrm{ng} / \mathrm{dL}$. A significant correlation was also observed between dissection types and mortality, as well as extravasation state. Conclusions: This preliminary study demonstrated that plasma SCUBE-1 level is a better and specific biomarker for AD and may be used for diagnosing $\mathrm{AD}$ in emergency services. Wider case series and further clinical studies are required to confirm these findings.
\end{abstract}

\section{Keywords}

SCUBE-1, Aortic dissection, Emergency service

\section{Introduction}

Aortic dissection (AD) is one of the troublesome diagnoses in emergency services. It requres rapid diagnosis and confirmation. The clinical picture beginning with chest pain has to be confirmed using imaging methods. However, the findings are always not clear, and patients can show only a simple finding imitating mere ischemia. Thus, it is possible that $\mathrm{AD}$ could be confused with findings suggesting renal colic, rendering the diagnosis a difficult issue. Ultrosonograpy and D-dimer level are also not always helpful. Contrast-induced and invasive tomography may not be applicable for all clinically suspected patients because of side effects and the cost [1]. Therefore, a helpful and noninvasive biomarker is necessary for clinicians to make a clear diagnosis.

Signal peptide-CUB (complement $\mathrm{C} 1 \mathrm{r} / \mathrm{C} 1 \mathrm{~s}$, Uegf, and Bmp1)-EGF (epidermal growth factor) domain-containing protein 1 (SCUBE-1) is a surface protein secreted during embryogenesis. In recent years, it was demonstrated that this protein can be used for multiple diagnoses, and there are also ongoing studies investigating SCUBE-1.

In the present study, we investigated whether SCUBE-1 protein could be useful for clinicians as a biomarker in the diagnosis of $\mathrm{AD}$.

\section{Materials and methods}

\subsection{Study setting}

This investigation was a prospective study conducted between January 01, 2019, and January 01, 2020. We included 20 patients diagnosed with $\mathrm{AD}$ who applied to the emergency department of our university hospital and 20 healthy volunteers. SCUBE-1 levels were assessed for both groups of subjects. Patients and volunteers were aged $\geq 18$ years. Twenty 
healthy subjects as a control group of a similar age as that of the AD group were also included. The control group was formed according to the inclusion and exclusion criteria and the average age of patients. Volunteers with known past medical history and no history of disease/surgery were included in this group.

\subsection{Study population}

This study was conducted in the emergency service of Izmir Katip Çelebi University and Research Hospital, Izmir, where an average of 700 patients apply daily. It is a 1000-bed hospital and one of the largest in this region. All health conditions requiring critical care, such as AD and cerebrovascular infarcts, are managed and facilities such as coronary angiography units are served at this hospital. It is the only critical care center in this region.

Patients who were considered to have $\mathrm{AD}$ or equivalent findings were included in this study. Those aged $\geq 18$ years, pregnant women, and those with a previous $\mathrm{AD}$ diagnosis were excluded. In addition, patients who had primary renal or hematological, severe hepatic, renal, or heart failure, as well as those who had been diagnosed with ischemic conditions such as mesenteric ischemia, were excluded. Patients allergic to contrast agent and those who had or still have a malignant tumor were also excluded. A medical history of a congenital diagnosis of a connective tissue disease such as Marfan syndrome was also considered as an exclusion criterion. Finally, patients who were not aware of their medical history were also excluded.

Patients who were admitted to the hospital with constant shock and vital imbalance were also not included as their statements could not be taken clearly and iatrogenic AD may occur during the procedures.

\subsection{Data collection}

Patients who were diagnosed with $\mathrm{AD}$ and consented to be a participant were primarily taken into the safety circle. Next, their blood samples were collected and analyzed at our hospital's laboratory using model/brand of Spectramax Plus 384 Elisa. The samples were analyzed using an enzyme-linked immunosorbent assay kit (catalog no. E-EL-H5405, Elabscience, Houston, United States) according to the manufacturer's instructions. The acquired data were recorded on forms prepared for patients.

Contrast-enhanced thoracoabdominal computed tomography (CT) angiography was performed for diagnostic purposes in the emergency department due to its high sensitivity and specificity in the diagnosis of AD. Diagnosis was performed using 128 slices of CT scan (Siemens Sonatom Definion AS 128 ) if the doctor thinks it is necessary. $350 / 100 \mathrm{~mL}$ iodideequivalent $755 \mathrm{~mL}$ of a nonionic omnipac iodinated contrast medium was injected intravenously at the rate of $60-70 \mathrm{~mL}$. $0.5 \mathrm{~mL} / \mathrm{s}$ and $30-40 \mathrm{~s}$ delay in screening. It is believed that the tomography scans of patients with clear images of all vascular structures are compatible for the study, including the aortic diameter, contour irregularities, intimal flaps, contrast extravasation, and enlargement of aneurysms for acute $\mathrm{AD}$ diagnosis.
Blood samples $(2 \mathrm{~mL})$ were collected from the patients and the control group in blood collection tubes, blood samples taken were centrifuged at $4000 \mathrm{rpm}$ for 10 minutes, and then stored in a freezer at $-80^{\circ} \mathrm{C}$ until analysis.

\subsection{Statistical analyses}

Statistical analyses were conducted using the SPSS 23.0 program for Windows ${ }^{\circledR}$ (IBM Inc., Chicago, IL, USA). Descriptive data were presented as number, percentage, mean, and standard deviation, median, minimum, and maximum values. The Kolmogorov-Smirnov test was used to evaluate the coherence of normal distribution of data. Pearson's chi-square and Fisher's exact tests were used for comparing categorical data. Independent T-test was conducted to compare two independent numerical values, and the Kruskal-Wallis test was used for comparing two different or three different numerical values. Results were considered to be significant at $\mathrm{p}<0.05$ with $95 \%$ confidence intervals.

\section{Results}

Among the study patients, there were 7 males and 13 females. Among the healthy volunteers, $50 \%(\mathrm{n}=10)$ were males and $50 \%(\mathrm{n}=10)$ were females. The demographic characteristics of both groups were similar with no statistical differences ( $p$ $>0.05)$. The average duration between the first arrival of patients to the emergency department and the diagnosis was $61.30 \pm 75.13$ (range $7-310$ ) min, whereas the average time for the arrival of the cardiovascular surgeon was $33.35 \pm 15.89$ (range 10-65) $\mathrm{min}$. The average hospitalization duration was $4.60 \pm 3.63$ (range $0-10$ ) days. Table 1 shows the information regarding the arrival features of patients, vital signs, and their demographic characteristics, including a summary of their diagnosis.

The outcomes of patients were analyzed according to the types of $\mathrm{AD}$, and no statistical significance was found between AD types and mortality. Type $1 \mathrm{AD}$ cases had a statistically significantly high mortality rate $(\mathrm{p}<0.001)$. We also analyzed the correlation between AD types and extravasation state and detected a statistically significantly high relationship in $\mathrm{AD}$ type 1 cases $(p<0.001)$. In addition, mortality rate was significantly higher in cases with extravasation $(\mathrm{p}<0.001)$ (Table 2).

The overall mean SCUBE-1 level of the study subjects was $16.11 \pm 5.33$ (range $7.26-27.84$ ) $\mathrm{ng} / \mathrm{dL}$, with $24.51 \pm 3.01$ $\mathrm{ng} / \mathrm{dL}$ in the patient group and $12.11 \pm 5.31 \mathrm{ng} / \mathrm{dL}$ in the control group. The mean SCUBE-1 level in the patient group was found to be significantly higher than that in the control group $(\mathrm{p}<0.001)$. A receiver-operating characteristic $(\mathrm{ROC})$ curve was plotted to determine the specificity and sensitivity of AD diagnosis (with 95\% confidence intervals) (Fig. 1), which demonstrated a sensitivity of $95 \%$ and a specificity of $76 \%$ when the SCUBE-1 level was $>19.75 \mathrm{ng} / \mathrm{dL}$. On the other hand, a significant correlation was found between AD types and mortality and the extravasation state. However, there were no significant differences in the mean SCUBE-1 levels based on the AD types, outcomes, and extravasation $(p>0.05)$ (Table 3). 
TA B L E 1. Details about the cases.

\begin{tabular}{|lc|}
\hline Parameter & $\begin{array}{c}\text { n (\%) or Average } \\
(\text { IQR or } \pm \text { SS) }\end{array}$ \\
\hline Age & $58.35 \pm 16.97$ \\
\hline Male & $65.35 \pm 18.62$ \\
\hline Female & \\
\hline Gender & $23(57.5)$ \\
\hline Male & $17(42.5)$ \\
\hline Female & \\
\hline Admission Complaint & $14(70.0)$ \\
\hline Chest Pain & $3(15.0)$ \\
\hline Back Pain & $3(15.0)$ \\
\hline Other &
\end{tabular}

Admission Vital Findings

\begin{tabular}{|lc}
\hline Pulse (/Min) & $93.60 \pm 18.72$ \\
\hline Systolic Blood Pressure $(\mathrm{mmHg})$ & $127.25 \pm 29.74$ \\
\hline Diastolic Blood Pressure $(\mathrm{mmHg})$ & $64.42 \pm 16.28$ \\
\hline Aortic Dissection Types &
\end{tabular}

Aortic Dissection Types

\begin{tabular}{|ll} 
Type I & $8(40.0)$ \\
\hline Type II & $3(15,0)$ \\
Type III & $9(45.0)$
\end{tabular}

\section{Extravasation Status}

\begin{tabular}{lc} 
Positive & $6(30.0)$ \\
\hline Negative & $14(70.0)$ \\
Outcome & $10(50.0)$ \\
\hline Discharge & $10(50.0)$ \\
\hline Exitus & \\
\hline HT Status & $13(65.0)$ \\
Positive & $7(35.0)$ \\
\hline Negative & \\
\hline CPR* in Emergency Room & $3(15.0)$ \\
\hline Positive & $17(85.0)$ \\
\hline Negative & \\
\hline Treatment & $2(10.0)$ \\
\hline Medical Treatment & $5(25.0)$ \\
\hline Preoperative Exitus & $8(40.0)$ \\
\hline Postopertive Discharge & $5(25.0)$ \\
\hline Postoperative Exitus & \\
\hline
\end{tabular}

* CPR: Cardiopulmonary resuscitation.

\section{Discussion}

AD comprises the most serious and a life-threatening situation. Although not known clearly, the frequency of $\mathrm{AD}$ has been predicted to be $5-30$ cases in a million population. The diagnosis of AD in emergency services remains a challenging issue. Especially, patients with a history of hypertension are at risk in this context. In the present study, a history of HT was present in $65 \%$ of the cases $(n=13)$. Contrary to

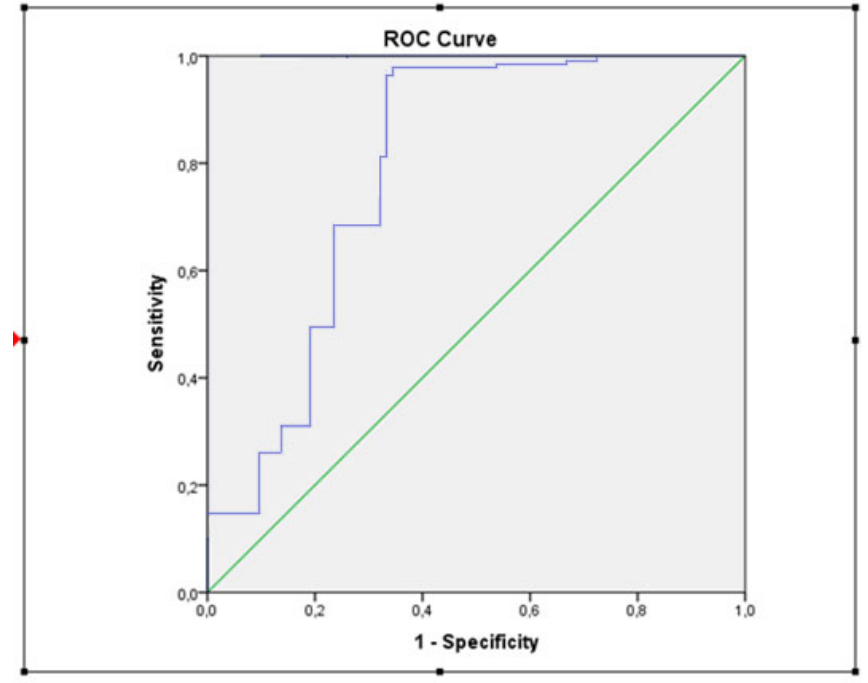

F I G U R E 1. ROC analysis of subjects' SCUBE-1 levels. 95\% sensitivity and 76\% specificity were determined on the cut-off value calculated for $A D$. AUC $=0.863(95 \% C I=0.70$ 1). $A U C=$ Area under the curve; $A D=$ Aortic dissection; $R O C$ = Receiver-operating characteristic.

TA B L E 2. Diagnosis/outcome status of cases in the Patient Group.

\begin{tabular}{|c|c|c|c|}
\hline \multicolumn{4}{|c|}{ Outcome } \\
\hline Dissection Types & Exitus & Discharge & $\mathbf{p}^{*}$ \\
\hline Type I & $7(87.5)$ & $1(12.5)$ & \\
\hline Type II & $1(33.3)$ & $2(66.6)$ & $<0.001$ \\
\hline Type III & $2(22.2)$ & $7(77.8)$ & \\
\hline \multicolumn{4}{|l|}{ Extravasation } \\
\hline Positive & $6(42.9)$ & $8(57.1)$ & \\
\hline Negative & $4(66.7)$ & $2(33.3)$ & $<\mathbf{0 . 0 0 1}$ \\
\hline \multicolumn{4}{|c|}{ Extravasation } \\
\hline Dissection Types & Positive & Negative & \\
\hline Type I & $6(75.0)$ & $2(25.0)$ & \\
\hline Type II & $2(66.6)$ & $1(33.3)$ & $<\mathbf{0 . 0 0 1}$ \\
\hline Type III & $6(66.7)$ & $3(33.3)$ & \\
\hline
\end{tabular}

the general belief that atypical symptoms are quite common, more than one-third of patients have findings and symptoms secondary to involvement of organ systems. When atypical symptoms are not recognized, potential death is inevitable. These symptoms include $50 \%$ pulse deficits, $20 \%$ neurological deficits, $5 \%$ syncope, and $5 \%-10 \%$ painlessness $[1,2]$. Diagnosis is made using tests such as contrast-enhanced CT angiography, which are expensive and have risks due to radiation and contrast agent. Nonetheless, this test remains the most commonly used diagnostic tool for AD. Hence, diagnostic tests that are inexpensive, noninvasive, and with less risk of complication are necessary. Although D-dimer is a supportive marker for $\mathrm{AD}$, age $>70$ years, pregnancy, active malignancy or metastasis, recent surgeries, trauma, deep vein 
TA B L E 3. Estimated mean SCUBE-1 values.

\begin{tabular}{lcc} 
Parameter & SCUBE-1 Level (ng/dL) & p \\
Groups & & \\
Patient Group & $24.51 \pm 3.01$ & $<\mathbf{0 . 0 0 1 *}$ \\
Control Group & $12.11 \pm 5.31$ & \\
Aortic Dissection Types & & \\
Type I & $25.32 \pm 3.33$ & $0.197 * *$ \\
Type II & $26.44 \pm 2.04$ & \\
Type III & $23.14 \pm 2.58$ & \\
Extravasation Status & & \\
Positive & $25.45 \pm 1.67$ & \\
Negative & $24.10 \pm 3.41$ & \\
Outcome & & \\
\hline Discharge & $24.39 \pm 3.80$ & \\
\hline Exitus & $24.62 \pm 2.17$ & \\
\hline
\end{tabular}

*: Independent T-Test; **: Kruskal-Wallis test was used.

thrombosis, hepatic failure, rheumatoid arthritis, and presence of infection are potential causes of false-positives. In case of warfarin treatment, the presence of small and isolated thrombi and having symptoms lasting longer than 5 days with falsenegative outcomes are detected [2]. Furthermore, D-dimer causes confusion for AD diagnosis, and there are also reports of possible D-dimer negativity in patients with $\mathrm{AD}$ [3]. There are also reports of patients with negative D-dimer levels but being operated [4]. Ultrasonography (USG) is also a contributory test for the diagnosis. However, emergency physicians have limited experience in this regard. Reliability of USG is variable because it may provide different results depending on the characteristics of patients and the physician experience. A noninvasive and safe diagnostic method useful for making the diagnosis is unavailable, indicating the crucial need for clinical studies.

SCUBE-1 is a cell surface protein released during embryogenesis. Its family members consist of amino-terminal signal peptide, repeated nine copies of EGF-like $\mathrm{N}$ terminal, spacer, CUB-domain signal peptide $\mathrm{C}$ terminal sequence, and structural cysteine molecules [5]. This protein is expressed on thrombocytes and vascular endothelial cells. It is involved in thrombosis by taking part in the structure of EGF glycoprotein. It also plays a role in thrombin activation and inflammation [6]. In case of vascular damage, there is an increase in the expression of SCUBE-1.

Acipayam et al. study reported that SCUBE-1can indicate early stages of thrombosis in patients with beta thalassemia [7]. In recent years, this protein has been investigated because of its role in vascular damage and thrombosis. Dia et al. demonstrated that SCUBE-1 expression can be detected $6 \mathrm{~h}$ after cardiac ischemia (SCUBE-1 level in patients with ACS: $205 \mathrm{ng} / \mathrm{mL}$ ), and it has low sensitivity and is a good marker for thrombosis (In our study, the SCUBE-1 level was measured as $27.84 \mathrm{ng} / \mathrm{mL}$ in the majority of AD cases.). In the same study, the authors stated that it is a reliable marker for diagnosing ischemic stroke [8]. Moreover, it has been demonstrated that its expression can be detected within $2 \mathrm{~h}$ in cases of mesenteric ischemia [9]. A specific biomarker for pulmonary emboli (PE) is also lacking. In this context, in a study conducted by Türkmen et al., it was reported that SCUBE-1 can be used for PE diagnosis as well [10].

$\mathrm{AD}$ is a pathology manifested by vascular endothelial damage and thrombi. SCUBE-1 protein is produced from the vascular endothelium, and its levels in the blood increase in cases of vascular structure damage and thrombi [4]. We observed SCUBE-1 levels can increase in AD as well, in our study. This unexpected increase would support the clinical predictivity. A recommended biomarker for $\mathrm{AD}$ is lacking, and although determining D-dimer levels is recommended, its diagnostic value is low. Several studies have stated not to use D-dimer positive and negative values for diagnosis, although there are meta-analyses that have indicated using its negative predictive value [1]. In the present study, we determined the AUC value of SCUBE-1 for AD diagnosis as 0.863 , in addition to $95 \%$ sensitivity and $76 \%$ specificity when the SCUBE- 1 cutoff level was $19.75 \mathrm{ng} / \mathrm{dL}$. Based on our study results and in comparison with clinical findings, we believe that SCUBE-1 would be a helpful biomarker for clinicians for diagnosing AD.

\section{Conclusion}

$\mathrm{AD}$ is a diagnosis associated with a high mortality rate in emergency services. Therefore, scientific studies are important to help in the diagnosis. We observed that plasma SCUBE-1 levels have high specificity for AD diagnosis and thus can be valuable. We suggest further studies with larger clinical series to support the resuls of our preliminary study.

\section{LIMITATIONS}

The first limitation of our study was the relatively small numbers of patients and controls, although these numbers were predicted by a power analysis. Next, the average age of the subjects was high in our study, as it is known that AD can also present in younger patients. Finally, according to the manufacturer information on the analysis kit, plasma samples cannot be stored in freezers for more than 2 months. This storage period can be a limitation, although the duration was not expired in our study.

\section{ACKNOWLEDGMENTS}

We are grateful to Assoc. Prof. Sadık Volkan Emren who works as cardiologist in our hospital for his contributions.

\section{CONFLICT OF INTEREST}

None of the authors received any type of financial support that could be considered potential conflict of interest regarding the manuscript or its submission. 


\section{ETHICAL CONSIDERATION}

Approval for conducting the study was obtained from the ethical committee of the university hospital (Izmir Katip Celebi University Clinical Studies Ethics Committee). Ethical committee number: 2019-KAE-0252. Consent forms were obtained from all patients and healthy volunteers or their firstdegree relatives. The entire study was conducted according to the criteria of the Declaration of Helsinki.

\section{REFERENCES}

[1] Johnson LouiseGA, Prince A. Aortic dissection and related aortic syndromes. In: Tintinalli JE, Stapczynski JS, Ma JO (editors). Tintinalli's Emergency Medicine: A Comprehensive Study Guide, 8th ed. New York, NY: McGraw-Hill Companies Inc. 2016:412-416.

[2] Kline JA. Thromboembolism. In: Tintinalli JE, Stapczynski JS, Ma JO (editors). Tintinalli's Emergency Medicine: A Comprehensive Study Guide, 8th ed. New York, NY: The McGraw-Hill Companies Inc. 2016:388-8.

[3] Nitta K, Imamura H, Kashima Y, et al. Impact of a negative D-dimer result on the initial assessment of acute aortic dissection. Int J Cardiol. 2018;258:232-236.

[4] Nitta K, Imamura H, Kashima Y, et al. Response to letter from Jiang: The diagnostic value of combined D-dimer with other indicators in suspected acute aortic dissection patients. Int J Cardiol. 2018;267:196.
[5] Tu CF, Su YH, Huang YN, et al. Localization and characterization of a novel secreted protein SCUBE1 in human platelets. Cardiovasc Res. 2006;71:486-495.

[6] Yang RB, Ng CKD, Wasserman SM, et al. Identification of a novel family of cell-surface proteins expressed in human vascular endothelium. J Biol Chem. 2002;277:46364-46373.

[7] Acipayam C, Tuncel DA, Güneş H, et al. Investigation Of Scube-1 Levels İn Pediatric Patients With Beta-Thalassemia. J Surg Med. 2019;3:825828.

[8] Dai DF, Thajeb P, Tu C-F, et al. Plasma concentration of SCUBE1, a novel platelet protein, is elevated in patients with acute coronary syndrome and ischemic stroke. Am College Cardiol. 2008;51:2173-2180.

[9] Turkmen S, Mentese S, Mentese A, et al. The value of signal peptideCUB-EGF domain-containing protein 1 and oxidative stress parameters in the diagnosis of acute mesenteric ischemia. Acad Emergency Med. 2013;20:257-264.

[10] Turkmen S, Sahin A, Gunaydin M , et al. The value of signal peptide-cub-egf domain-containing protein-1 (SCUBE1) in the diagnosis of pulmonary embolism: a preliminary study. Acad Emerg Med. 2015;22:922-926.

How to cite this article:Adem Çakır, Umut Payza, Saliha Aksun, Ahmet Kayalı, Zeynep Karakaya, Fatih Esad Topal. Validity of Signal Peptide-CUB-EGF Domain-containing Protein-1 (SCUBE-1) in the Diagnosis of Aortic Dissection. Signa Vitae. 2021;17(1):112-116. doi:10.22514/sv.2020.16.0043. 